\title{
COSMOLOGICAL STUDIES WITH RADIO GALAXIES AND SUPERNOVAE
}

\author{
Ruth A. Daly ${ }^{1}$, Matthew P. Mory ${ }^{1}$, C. P. O’Dea ${ }^{2}$, P. Kharb ${ }^{2}$, S. Baum ${ }^{2}$, E. J. Guerra ${ }^{3}$, and S. G. Djorgovski ${ }^{4}$ \\ ${ }^{1}$ Department of Physics, Penn State University, Berks Campus, P.O. Box 7009, Reading, PA 19610, USA; rdaly@psu.edu \\ ${ }^{2}$ Rochester Institute of Technology, 54 Lomb Memorial Drive, Rochester, NY 14623, USA \\ ${ }^{3}$ Department of Physics and Astronomy, Rowan University, 201 Mullica Hill Rd. Glassboro, NJ 08028, USA \\ ${ }^{4}$ Division of Physics, Mathematics, and Astronomy, California Institute of Technology, MS 105-24, Pasadena, CA 91125, USA \\ Received 2007 May 18; accepted 2007 October 14; published 2009 January 28
}

\begin{abstract}
Physical sizes of extended radio galaxies can be employed as a cosmological "standard ruler," using a previously developed method. Eleven new radio galaxies are added to our previous sample of 19 sources, forming a sample of 30 objects with redshifts between 0 and 1.8. This sample of radio galaxies are used to obtain the best-fit cosmological parameters in a quintessence model in a spatially flat universe, a cosmological constant model that allows for nonzero space curvature, and a rolling scalar field model in a spatially flat universe. Results obtained with radio galaxies are compared with those obtained with different supernova samples, and with combined radio galaxy and supernova samples. Results obtained with different samples are consistent, suggesting that neither method is seriously affected by systematic errors. Best-fit radio galaxy and supernovae model parameters determined in the different cosmological models are nearly identical, and are used to determine dimensionless coordinate distances to supernovae and radio galaxies, and distance moduli to the radio galaxies. The distance moduli to the radio galaxies can be combined with supernovae samples to increase the number of sources, particularly highredshift sources, in the samples. The constraints obtained here with the combined radio galaxy plus supernovae dataset in the rolling scalar field model are quite strong. The best-fit parameter values suggest that $\Omega_{m}$ is less than about 0.35 , and the model parameter $\alpha$ is close to zero; that is, a cosmological constant provides a good description of the data. We also obtain new constraints on the physics of engines that power the large-scale radio emission. The equation that describes the predicted size of each radio source is controlled by one model parameter, $\beta$, which parameterizes the extraction of energy from the black hole. Joint fits of radio galaxy and supernova samples indicate a best-fit value of $\beta$ that is very close to a special value for which the relationship between the braking magnetic field strength and the properties of the spinning black hole is greatly simplified, and the braking magnetic field strength depends only upon the spin angular momentum per unit mass and the gravitational radius of the black hole. The best-fit value of $\beta$ of 1.5 indicates that the beam power $L_{j}$ and the initial spin energy of the black hole $E$ are related by $L_{j} \propto E^{2}$, and that the relationship that might naively be expected for an Eddington limited system, $L_{j} \propto E$, is quite clearly ruled out for the jets in these systems.

Key words: cosmological parameters - cosmology: observations - cosmology: theory - dark matter - equation of state
\end{abstract}

\section{INTRODUCTION}

Recent cosmological studies using cosmic microwave background radiation (CMBR), supernovae, and other types of astronomical sources and phenomena have greatly improved our understanding of the recent expansion and acceleration history of the universe. Whereas a consistent picture has emerged (the "concordance cosmology"), in which the dynamics of the universe is currently dominated by a mysterious dark energy, its physical nature remains one of the key outstanding problems of physical science today. For a summary of developments in this field see Ratra \& Vogeley (2008).

Aside from the CMBR, most experimental methods to study the expansion history of the universe, and thus its matterenergy contents, require samples of standardizable sources whose distances can be determined in a consistent way, e.g., the supernovae of Type Ia. It is clear that both low- and high redshift sources play an important role in these studies. Lowto-moderate-redshift sources allow us to define and probe the acceleration of the universe and the properties of the dark energy at the current epoch, whereas higher redshift sources allow us to probe the properties of the dark energy at earlier epochs and possible changes in its properties, which is perhaps the best path towards understanding its physical nature. Confidence in luminosity and coordinate distance determinations, which are the foundation of the studies discussed here, is bolstered when more than one method yields the same results.

Like supernovae, powerful radio galaxies are observed out to redshifts greater than one, and their observable properties can be used to determine the coordinate distances to them, or equivalently the luminosity distances or distance moduli (Daly 1994). It is thus interesting to study these sources and to determine whether cosmological results obtained with radio galaxies agree with those obtained with supernovae and other methods. In addition, the radio galaxy and supernova samples may be analyzed jointly to improve the determinations of the radio galaxy model parameters.

In this paper, 11 new radio galaxies are combined with a previously studied sample of 19 sources, to yield a sample of 30 radio galaxies suitable for cosmological studies. This sample is analyzed here in three cosmological models. The first model allows for quintessence and nonrelativistic matter in a spatially flat universe; the second model allows for nonrelativistic matter, a cosmological constant, and space curvature; and the third model allows for a rolling scalar field in a spatially flat universe (Ratra \& Peebles 1988). All of these models are based on the equations of general relativity, and rely upon this as the correct theory of gravity. 
The primary objectives are to obtain and compare constraints on model and cosmological parameters using different samples of Type Ia supernovae, extended radio galaxies, and a combined sample of supernovae and radio galaxies. Similar results obtained with the radio galaxy and supernovae methods, which determine similar quantities over similar redshifts, will bolster our confidence in each method. This is because the methods rely upon measurements of entirely different quantities that are then applied using completely different astrophysical arguments, and thus provide independent measures of coordinate distances and cosmological parameters. Results obtained from the combined sample allow strong constraints to be placed on the radio galaxy model parameter, which provides a direct link to and diagnostic of the physics of the energy extraction that produces the large-scale jets in these systems. Finally, if very similar best-fit model parameters are obtained in different cosmological scenarios, tben these parameters can be used to determine the dimensionless coordinate distance to each source. The dimensionless coordinate distances thus obtained are then used for a separate study. In addition, the dimensionless coordinate distances to the radio galaxies can be used to define the distance modulus to each radio galaxy, which can be combined with those of supernovae to increase the sample sizes.

The use of powerful extended radio galaxies as a cosmological tool is reviewed in Section 2. Results obtained with radio galaxies, supernovae, and combined samples of radio galaxies and supernovae are presented in Section 3. Determinations of distance moduli to radio galaxies are presented in Section 4. The main results and conclusions of the paper are summarized in Section 5.

\section{RADIO GALAXIES AND COSMOLOGY}

Powerful radio sources are a cosmological population which can be observed out to very high redshifts, reaching beyond the practical limits of supernova studies. This makes them in principle a very interesting cosmological probe, provided that some distance-dependent quantity such as physical size can be standardized. In Section 2, we describe how the maximum size that a radio source will reach during its lifetime, $2 D_{*}$, can be determined from radio observations.

There are many different types of radio sources such as compact and extended radio sources, and radio galaxies and radio loud quasars. The use of radio sources for cosmological studies has a long and distinguished history including the works of Rowan-Robinson (1967); Longair \& Pooley (1969); Hoyle \& Burbidge (1970); Kellerman (1972, 1993); Fanaroff \& Longair (1972); Kapahi (1975, 1985); Rees (1972); Readhead \& Longair (1975); Longair (1976); Wall et al. (1980); Laing et al. (1983); Condon (1984a, 1984b); Gopal-Krishna \& Wiita (1987); Pelletier \& Roland (1989); Dunlop \& Peacock (1990); Singal (1993); Daly (1994); Kayser (1995); Buchalter et al. (1998); Guerra \& Daly (1998); Kaiser \& Alexander (1999); Gurvits et al. (1999); Guerra et al. (2000); Rawlings (2002); Daly \& Guerra (2002); Chen \& Ratra (2003); Podariu et al. (2003); Jamrozy (2004); Jackson (2004); Barai \& Wiita (2006, 2007), and Jackson \& Jammetta (2006).

Here, very powerful radio galaxies are used as a modified standard yardstick for cosmological studies. Radio galaxies rather than radio loud quasars are selected for study so as to minimize projection effects; in the standard unified model for radio galaxies and radio loud quasars, the sources are intrinsically the same, but radio galaxies lie close to the plane of the sky, and radio loud quasars are oriented along the line of sight to the observer.
The subset of classical double radio galaxies that are very powerful form a very homogeneous population. The source properties and structure are well described by the standard "twin jet" model (e.g. Blandford and Rees 1974; Scheuer 1974; Begelman et al. 1984; Begelman \& Cioffi 1989; Daly 1990; Leahy 1991). Particles are accelerated and material is channeled away from the vicinity of a massive black hole along relatively narrow, oppositely directed jets, and is deposited in the radio hot spot. Here particles are re-accelerated to relativistic energies and produce synchrotron radiation in the presence of a local magnetic field. Relativistic plasma flows from the radio hot spots, and, as time goes on, the location of the radio hot spot moves further from the central black hole leaving behind a "radio bridge" or "radio lobe" of relativistic material.

\subsection{Overview of the Extended Radio Galaxy (ERG) Method}

The ERG method considered here was proposed by Daly (1994), and explored and applied by Guerra \& Daly (1998); Guerra et al. (2000); Daly \& Guerra (2002), and Podariu et al. (2003). It is described in detail in these papers, and is summarized briefly here.

The method is based upon the following observations. (1) The most powerful extended classical double radio galaxies, those with $178 \mathrm{MHz}$ radio powers greater than about $\left(3 \times 10^{26}\right) h^{-2} \mathrm{~W} \mathrm{~Hz}^{-1} \mathrm{sr}^{-1}$, have very regular radio bridge structure and shape (e.g. Leahy et al. 1989). This indicates that the sources are growing at a rate that is well into the supersonic regime, and that there is minimal backflow of material within the radio bridge (Leahy \& Williams 1984; Alexander 1987; Alexander \& Leahy 1987; Leahy et al. 1989). Thus, strong shock physics, which is clean and simple, can be applied to these systems. It also indicates that the velocity deduced by studying a source represents the average rate of growth of the source, that is, corrections for backflow of material within the bridge are negligible. (2) The average size of this special class of powerful classical double radio galaxies at a given redshift $\langle D\rangle$ has a small dispersion independent of the cosmological model, as illustrated in Figure 8 of Guerra et al. (2000). This means that $2\langle D\rangle$ provides a measure of the maximum size that a given source at that redshift will reach during its lifetime.

The parent population of radio galaxies considered for this study are from the complete sample of 3CRR radio galaxies with $178 \mathrm{MHz}$ radio powers greater than about $\left(3 \times 10^{26}\right) h^{-2} \mathrm{~W} \mathrm{~Hz}^{-1} \mathrm{sr}^{-1}$. This leads to a sample of 70 radio galaxies (Guerra \& Daly 1998). This parent population is used to define $\langle D\rangle$ in several redshift bins, as described by GD98. This measure of the mean or maximum size of a given source at similar redshift depends upon the cosmological model through the coordinate distance $\left(a_{o r}\right)$, since the size of a given source is $D \propto\left(a_{o} r\right)$.

Individual sources from this parent population are studied in detail to arrive at an independent measure of the average size of an individual source, $D_{*}$. It turns out that $D_{*} \propto\left(a_{o} r\right)^{4 / 7-2 \beta / 3}$, where $\beta$ is a model parameter to be determined. The ratio $R_{*} \equiv\langle D\rangle / D_{*}$ is approximately given by $R_{*} \propto\left(a_{o} r\right)^{3 / 7+2 \beta / 3}$, so requiring that this ratio remain constant allows determinations of both $\beta$ and the cosmological parameters that determine $\left(a_{o r}\right)$ to a particular source at redshift $\mathrm{z}$. When the fits are run, the full dependence of each factor that enters the ratio $R_{*}$ is, of course, included. The full equation for $D_{*}$ and the way that it was derived is described below (see also Daly 1990, 1994; GD98; GDW00; DG02). 
Each side of a source grows with an average velocity $v$ for a total time $t_{*}$, so a particular source will have an average size $D_{*}$ at the end of it's lifetime: $D_{*} \propto v t_{*}$. (Note that the source velocities are independent of source size, suggesting that the velocity of a given source is roughly constant over the lifetime of that source, as described in detail by O'Dea et al. 2008). The fact that the source sizes have a small dispersion at a given redshift suggests a cancelation between that factors that determine $v$ and those that determine $t_{*}$. The equations of strong shock physics indicate that the overall velocity $v$ with which the source lengthens is $v \propto\left[L_{j} /\left(n_{a} a_{L}^{2}\right)\right]^{1 / 3}$ (e.g. Rawlings \& Saunders 1991; Daly 1990), where $n_{a}$ is the ambient gas density and $a_{L}$ is the radius of the cross sectional area perpendicular to the direction of growth of the source. Thus, the average source size depends both of the intrinsic properties, $L_{j}$ and $t_{*}$, of the AGN that powers the outflow, and those that are extrinsic to the AGN, $n_{a}$ and $a_{L}^{2}$. Separating the intrinsic from the extrinsic factors we have $D_{*} \propto\left(L_{j}^{1 / 3} t_{*}\right)\left(n_{a} a_{L}^{2}\right)^{-1 / 3}$. As shown by Daly (1994), the extrinsic factors described by the second term have a very weak dependence on the coordinate distance $\left(a_{o r}\right)$ to the source, and go approximately as $k_{g} \propto\left(n_{a} a_{L}^{2}\right)^{-1 / 3} \propto\left(a_{o} r\right)^{-0.1}$. This parameter can be determined from observations by noting that $n_{a} \propto P / v^{2}$ (e.g., De Young 2002), so $k_{g} \equiv\left(P a_{L}^{2} / v^{2}\right)^{-1 / 3}$; here $P$ is the postshock pressure in the radio bridge $P=\left((4 / 3) b^{-1.5}+b^{2}\right)\left(B_{\min } / 24 \pi\right)$ and the parameter $b$ allows for offsets of the magnetic field strength $B$ from minimum energy conditions $B=b B_{\min }$.

Rearranging terms, we have

$$
D_{*} \propto v t_{*} \propto\left(L_{j}^{1 / 3} t_{*}\right) k_{g}
$$

To maintain the observed small dispersion in average source size $\langle D\rangle$, there must be a cancelation between the factors intrinsic to the AGN that affect the source size, $L_{j}$ and $t_{*}$. Thus, it is proposed that the total lifetime of the outflow $t_{*}$ be written as a power law in the beam power

$$
t_{*} \propto L_{j}^{-\beta / 3} .
$$

Then, the average size a given source would have if it were observed over its entire lifetime is $D_{*} \propto L_{j}^{(1-\beta) / 3}\left(P a_{L}^{2} / v^{2}\right)^{-1 / 3}$, or $D_{*} \propto L_{j}^{(1-\beta) / 3} k_{g}$. Thus, $D_{*}$ can be determined for an individual source; it has one model parameter, $\beta$, and depends upon the cosmological model through its depedence on $\left(a_{o r}\right)$.

This parameterization of the total time the AGN produces jets $t_{*} \propto L_{j}^{-\beta / 3}$ includes as a special case the relationship expected if the outflow is Eddington limited. For an Eddington limited system, the lifetime is independent of the beam power, so a value of $\beta=0$ is expected, and we will be able to test whether the outflows are Eddington limited.

The implications of the value of $\beta$ for physics quite close to the AGN are described by Daly \& Guerra (2002), and are discussed in more detail here in Section 3.1. The relationship $t_{*} \propto L_{j}^{-\beta / 3}$ fits quite nicely in standard magnetic braking models to power the outflows from AGN (e.g. Blandford 1990; see Section 3.1).

To write $D_{*}$ in terms of empirically determined quantities $a_{L}$, $v$, and $P$, we note that $D_{*} \propto v t_{*} \propto v L_{j}^{-\beta / 3}$ and $L_{j} \propto\left(v / k_{g}\right)^{3}$, so

$$
D_{*} \propto k_{g}^{\beta} v^{1-\beta} \propto\left(a_{L}^{2} P\right)^{-\beta / 3} v^{1-\beta / 3} .
$$

The dependence of $D_{*}$ on cosmological parameters enters through the dependence of each of the empirically determined quantities, $a_{L}, P$, and $v$, on the coordinate distance, and is approximately given by $D_{*} \propto\left(a_{o} r\right)^{4 / 7-2 \beta / 3}$ Daly (1994). Of course, when the full computation requiring that the ratio $\langle D\rangle / D_{*}$ remain constant is carried out, the full dependence of each quantity $a_{L}, P$, and $v$ on the coordinate distance is included.

\section{RESULTS}

Here, we consider three standard models: a quintessence model in a spatially flat universe; a lambda model that allows for nonzero space curvature, nonrelativistic matter, and a cosmological constant; and a rolling scalar field model in a spatially flat universe. The supernovae samples considered here are the 192 supernovae from Davis et al. (2007), the 182 supernovae sample of Riess et al. (2007), and the 115 supernovae sample of Astier et al. (2006). There is significant overlap between these samples; for example, the Davis et al. (2007) sample includes the essence data of Wood-Vasey (2007) and the high redshift data of Riess et al. (2007). Different samples are studied to be able to compare results obtained with each. In addition, the model parameters determined for each sample can be applied to that sample to solve for the dimensionless coordinate distances to the supernovae in that sample. The 30 radio galaxies studied here include the 11 new radio galaxies presented by Kharb et al. (2008), with details on individual source properties given by O'Dea et al. (2008), and the 19 radio galaxies previously studied by Guerra et al. (2000) and Daly \& Guerra (2002) with the source 3C427.1 removed, as discussed by Podariu et al. (2003).

The best-fit parameters obtained in these models are listed shown in Tables 1, 2, and 3. The fits are done in the standard way for radio galaxies and supernovae separately. For the radio galaxies, we use the relationship

$$
R_{*}=\langle D\rangle / D_{*}=k_{0} y^{(6 \beta-1) / 7}\left(k_{1} y^{-4 / 7}+k_{2}\right)^{\beta / 3-1},
$$

where the cosmological model enters through the determination of the dimensionless coordinate distance $y$ to each source, which is simply related to the coordinate distance $\left(a_{o r}\right), y=\left(H_{0} / c\right)\left(a_{o} r\right)$, and we have the standard equations $y=\int d z / E(z), E(z)=H(z) / H_{0}, H(z)=(1 / a)(d a / d t)$ is the expansion rate of the universe at a given redshift, and $k_{0}, k_{1}$, and $k_{2}$ are observed quantities (see Daly \& Djorgovski 2003 and the appendix of Daly \& Guerra 2002). We minimize the difference between $\ln \left(\langle D\rangle / D_{*}\right)$ and a constant, $\kappa_{\mathrm{RG}}$, as described in detail by Guerra \& Daly (1998), to obtain the best-fit values of $\beta$, $\kappa_{\mathrm{RG}}$, and cosmological parameters. The ratio $\langle D\rangle / D_{*}$ obtained for the best-fit values of cosmological parameters and $\beta$, and normalized to unity using the best-fit value of $\kappa_{\mathrm{RG}}$ is shown in Figure 1 for the quintessence model. As in Daly \& Guerra (2002), an offset from minimum energy conditions of $b=0.25$ has been adopted, and the results are insensitive to the value of $b$ assumed; similar results are obtained for $b=1$. For the supernovae, we use the relationship

$$
\mu=\kappa_{\mathrm{SN}}+5 \log 10[y(1+z)]
$$

where $\kappa_{\mathrm{SN}}$ is a constant to be fitted for each of the supernvae samples, and, as above, $y$ is the dimensionless coordinate distance, $y=\left(H_{0} / c\right)\left(a_{o} r\right)$. We minimize the difference between the observed and predicted values of $\mu$ to obtain the bestfit values for cosmological parameters and the constant $\kappa_{\mathrm{SN}}$. The parameter $\kappa_{\mathrm{SN}}$ can be related to the effective Hubble constant adopted by the supernovae group to obtain their values 
Table 1

Cosmological Results in a Quintessence Model

\begin{tabular}{lcccccc}
\hline \hline Sample & $w$ & $\Omega_{m}$ & $\beta$ & $\kappa_{\mathrm{SN}}$ & $\kappa_{\mathrm{RG}}$ & $\chi^{2} / \mathrm{dof}$ \\
\hline 30 RG & $-0.87_{-1.1}^{+0.3}$ & $0-0.25$ & $1.35 \pm 0.2$ & & $9.101 \pm 0.035$ & $28.9 / 26$ \\
192SN+30RG & $-1.08_{-.39}^{+.28}$ & $0.29_{-0.11}^{+0.08}$ & $1.52 \pm 0.15$ & $43.296 \pm 0.015$ & $9.035 \pm 0.035$ & $224.4 / 217$ \\
$192 \mathrm{SN}$ & $-1.14_{-0.4}^{+0.29}$ & $0.31_{-0.1}^{+0.8}$ & & $43.295 \pm 0.015$ & & $194.2 / 189$ \\
$182 \mathrm{SN}+30 \mathrm{RG}$ & $-1.66 \pm 0.66$ & $0.45_{-0.08}^{+0.05}$ & $1.55_{-0.1}^{+.15}$ & $43.356 \pm 0.016$ & $9.029 \pm 0.035$ & $186.8 / 207$ \\
$182 \mathrm{SN}$ & $-1.74 \pm 0.74$ & $0.46 \pm 0.06$ & & $43.354 \pm 0.016$ & & $155.7 / 179$ \\
$115 \mathrm{SN}+30 \mathrm{RG}$ & $-0.91_{-0.53}^{+0.33}$ & $0.22_{-0.22}^{+0.16}$ & $1.50 \pm 0.15$ & $43.158 \pm 0.015$ & $9.038 \pm 0.035$ & $142.6 / 140$ \\
$115 \mathrm{SN}$ & $-1.08_{-0.57}^{+0.44}$ & $0.29_{-0.26}^{+0.13}$ & & $43.154 \pm 0.015$ & & $112.5 / 112$ \\
\hline
\end{tabular}

Table 2

Cosmological Results in a Lambda Model with Space Curvature

\begin{tabular}{lcccccc}
\hline \hline Sample & $\Omega_{\Lambda}$ & $\Omega_{m}$ & $\beta$ & $\kappa_{\mathrm{SN}}$ & $\kappa_{\mathrm{RG}}$ & $\chi^{2} / \mathrm{dof}$ \\
\hline 30 RG & $0.85_{-1.3}^{+0.4}$ & $0-0.24$ & $1.35 \pm 0.2$ & & $9.112 \pm 0.035$ & $28.9 / 26$ \\
192SN+30RG & $0.8 \pm 0.18$ & $0.30 \pm 0.09$ & $1.52 \pm 0.15$ & $43.296 \pm 0.015$ & $9.034 \pm 0.035$ & $224.4 / 217$ \\
192SN & $0.85_{-0.19}^{+0.16}$ & $0.33_{-0.1}^{+0.08}$ & & $43.293 \pm 0.015$ & & $194.0 / 189$ \\
182SN+30RG & $0.91_{-0.2}^{+0.16}$ & $0.45 \pm 0.09$ & $1.60_{-0.15}^{+.1}$ & $43.365 \pm 0.016$ & $9.046 \pm 0.035$ & $186.9 / 207$ \\
182SN & $0.96_{-0.19}^{+0.16}$ & $0.48_{-0.09}^{+0.08}$ & & $43.362 \pm 0.016$ & & $155.6 / 179$ \\
115SN+30RG & $0.69_{-0.32}^{+0.25}$ & $0.22_{-0.22}^{+0.17}$ & $1.50 \pm 0.15$ & $43.157 \pm 0.015$ & $9.039 \pm 0.035$ & $142.6 / 140$ \\
115SN & $0.81_{-0.32}^{+0.28}$ & $0.31_{-0.22}^{+0.19}$ & & $43.153 \pm 0.015$ & & $112.5 / 112$ \\
& & & & & &
\end{tabular}

Table 3

Cosmological Results in a Rolling Scalar Field Model

\begin{tabular}{|c|c|c|c|c|c|c|}
\hline Sample & $\alpha$ & $\Omega_{m}$ & $\beta$ & $\kappa_{\mathrm{SN}}$ & $\kappa_{\mathrm{RG}}$ & $\chi^{2} /$ dof \\
\hline $30 \mathrm{RG}$ & $0-6.2$ & $0-0.25$ & $1.35_{-0.1}^{+0.2}$ & & $9.12 \pm 0.035$ & $29.1 / 26$ \\
\hline $192 \mathrm{SN}+30 \mathrm{RG}$ & $0_{-0}^{+1.25}$ & $0.27_{-0.12}^{+0.03}$ & $1.50_{-0.1}^{+0.15}$ & $43.301 \pm 0.015$ & $9.032 \pm 0.035$ & $224.5 / 217$ \\
\hline $192 \mathrm{SN}$ & $0_{-0}^{+0.95}$ & $0.27_{-0.1}^{+0.03}$ & & $43.304 \pm 0.015$ & & $194.3 / 189$ \\
\hline $182 \mathrm{SN}+30 \mathrm{RG}$ & $0_{-0}^{+0.5}$ & $0.34_{-0.07}^{+0.04}$ & $1.55_{-0.1}^{+0.15}$ & $43.394 \pm 0.016$ & $9.009 \pm 0.035$ & $188.5 / 207$ \\
\hline $182 \mathrm{SN}$ & $0_{-0}^{+0.45}$ & $0.34_{-0.06}^{+0.04}$ & & $43.394 \pm 0.016$ & & $157.9 / 179$ \\
\hline $115 \mathrm{SN}+30 \mathrm{RG}$ & $0.35_{-0.35}^{+3.7}$ & $0.21_{-0.15}^{+0.08}$ & $1.50 \pm 0.15$ & $43.158 \pm 0.015$ & $9.039 \pm 0.035$ & $142.6 / 140$ \\
\hline $115 \mathrm{SN}$ & $0_{-0}^{+3.85}$ & $0.26_{-0.21}^{+0.04}$ & & $43.156 \pm 0.015$ & & $112.6 / 112$ \\
\hline
\end{tabular}

of $\mu, \kappa_{\mathrm{SN}}=25-5 \log 10\left(H_{0} / c\right)$, where $H_{0}$ is in units of $\mathrm{km} / \mathrm{s} / \mathrm{Mpc}$, and $\mathrm{c}$ is in units of $\mathrm{km} / \mathrm{s}$. For example, a value of $H_{0}=70 \mathrm{~km} \mathrm{~s}^{-1} \mathrm{Mpc}^{-1}$ translates to a value of $\kappa_{\mathrm{SN}}$ of about 43.15. This approach is convenient since effective values of $H_{0}$ adopted and used in supernovae studies are often not included in publications and are often otherwise unavailable for the supernovae samples; the approach used here by-passes the need to know the specific value of $H_{0}$ adopted and applied to a particular supernovae sample. The exception is the supernovae sample of Astier et al. (2006) who adopted a value of $H_{0}=$ $70 \mathrm{~km} \mathrm{~s}^{-1} \mathrm{Mpc}^{-1}$, corresponding to a value of $\kappa_{\mathrm{SN}}=43.15$; the values we recover here (see Tables 1,2, and 3) are consistent with the input value of Astier et al. (2006). Once the best-fit value of $\kappa_{\mathrm{SN}}$ has been determined for a particular dataset, this value and the observed value of $\mu$ can be substituted into Equation (5) to solve for the dimensionless coordinate distance to the sources in that sample; the values thus obtained are listed in Daly et al. (2008). Similarly, once the best-fit values of the constants $\kappa_{\mathrm{RG}}$ and $\beta$ have been obtained, Equation (4) can be used to solve for the dimensionless coordinate distance $y$ to each radio galaxy. Then, Equation (5) can be used to define an effective distance modulus $\mu$ to each radio galaxy so that the radio galaxies can be added to and analyzed with the supernovae samples. Of course, in doing this, the best-fit value of $\kappa_{\mathrm{SN}}$ obtained for a particular supernovae sample is used to obtain values of $\mu$ for the radio galaxies that are to be added to that sample.

To obtain the best-fit parameters for the joint fits, the chisquare for each set of cosmological parameter and model parameter values is obtained by adding the chi-square obtained

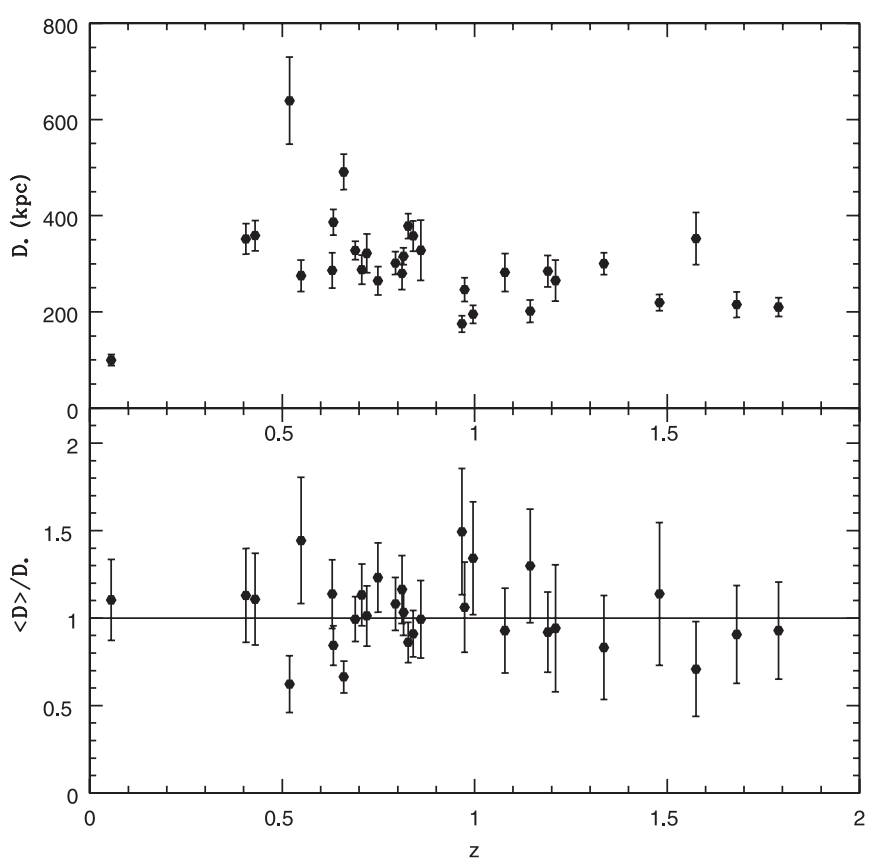

Figure 1. Values of $D_{*}$ and the ratio $\langle D\rangle / D_{*}$ for the 30 radio galaxies obtained using the the best-fit parameters of the quintessence model for radio galaxies alone and normalized using the best-fit value of $\kappa_{\mathrm{RG}}$.

for the radio galaxies and supernovae, and finding the minimum chi-square of the joint fit. That is, we require that a single 


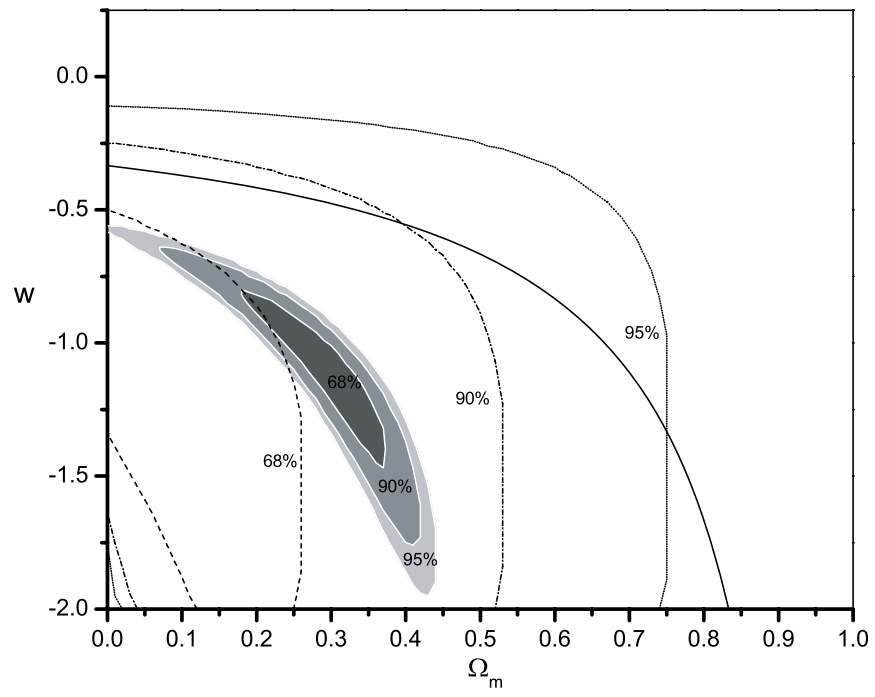

Figure 2. Constraints on the equation of state parameter $w$ and $\Omega_{m}$ obtained in a spatially flat quintessence model with 30 radio galaxies alone (dashed lines) overlayed with those obtained with the combined sample of 192 supernovae and 30 radio galaxies (solid contours). The solid line separates an accelerating from a decelerating universe; points below the line are parameter values for which the universe is accelerating at the current epoch in this model.

set of cosmological parameters and model parameters $\beta, \kappa_{\mathrm{RG}}$, and $\kappa_{\mathrm{SN}}$ describe both the radio galaxy and supernovae datasets simultaneously, and find the best-fit values of parameters that minimizes this total chi-square. This is quite helpful in constraining the model parameter $\beta$, as discussed below.

Each of the radio galaxy fits involve four parameters, two cosmological parameters and two model parameters $\left(\kappa_{\mathrm{RG}}\right.$ and $\beta$ ), or 26 degrees of freedom for 30 radio galaxies. The supernovae fits involve three parameters, two cosmological parameters and one model parameter $\left(\kappa_{\mathrm{SN}}\right)$, since the supernovae model parameter that relates the rate of decline of the light curve to the peak supernovae brightness is obtained separately. The joint supernovae and radio galaxy fits involve five parameters, two cosmological parameters and three model parameters $\left(\kappa_{\mathrm{SN}}\right.$, $\kappa_{\mathrm{RG}}$, and $\beta$ ). The reduced $\chi^{2}$ for the best-fit parameters is of order unity for radio galaxy sample alone, for most of the supernovae samples alone, and for the joint supernovae and radio galaxy fits, indicating that the model provides a good description of the data for the best-fit parameters listed, though the reduced $\chi^{2}$ obtained for the 182 supernovae is a bit low. Values of cosmological parameters obtained here are consistent with those obtained and published by each of the supernovae groups. In addition to the best-fit values of cosmological parameters, we also list the bestfit values of the model parameters, $\kappa_{\mathrm{SN}}, \kappa_{\mathrm{RG}}$, and $\beta$, which are needed to solve for the dimensionless coordinate distance to each source. It is also interesting to compare best-fit parameter values obtained with radio galaxies alone, supernovae alone, and the combined radio galaxy and supernovae samples.

Values of $D_{*}$ and the ratio $\langle D\rangle / D_{*}$ are shown in Figure 1 for the best-fit parameters obtained with the 30 radio galaxies alone in the quintessence model (see Table 2), normalized using the best-fit value of $\kappa_{\mathrm{RG}}$ to have a value of unity. The values of $D_{*}$ clearly change with redshift as expected; they should change with redshift in the same way as $\langle D\rangle$, which is shown in Figure 8 of Guerra et al. (2000). The ratio $\langle D\rangle / D_{*}$ is independent of redshift, as predicted in the model.

Results obtained in a quintessence model assuming a spatially flat universe are shown in Figures 2-5. Results obtained with

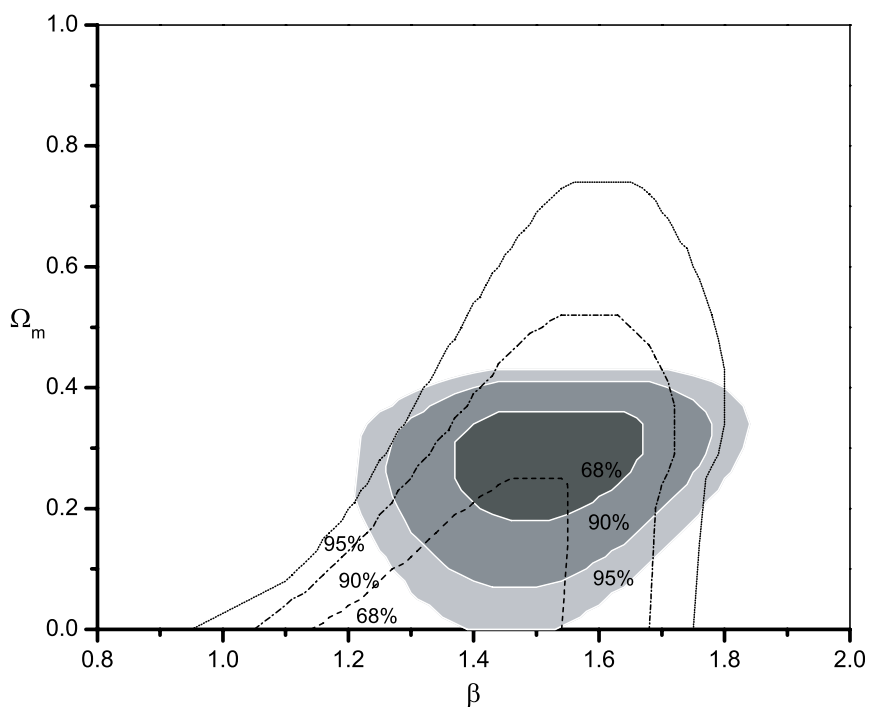

Figure 3. As in Figure 2 for the parameters $\beta$ and $\Omega_{m}$.

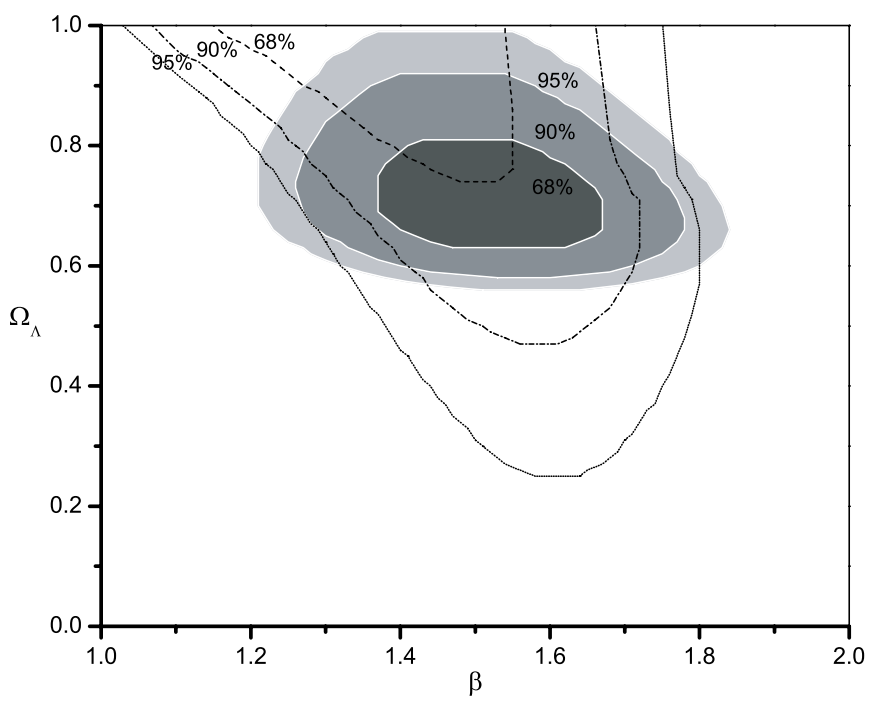

Figure 4. As in Figure 2 for the parameters $\beta$ and $\Omega_{\Lambda}$.

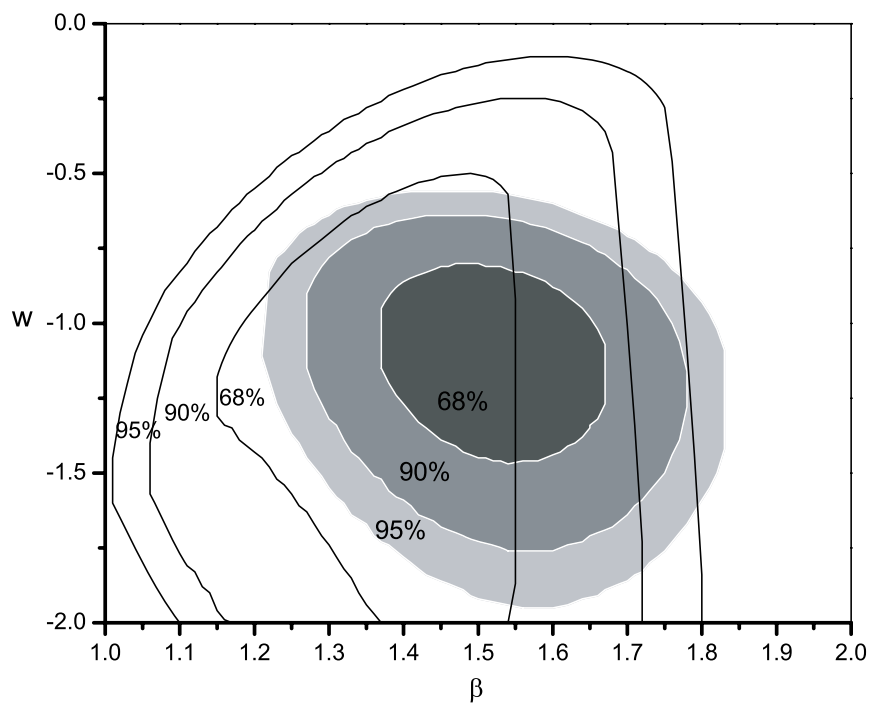

Figure 5. As in Figure 2 for the parameters $\beta$ and $w$. 


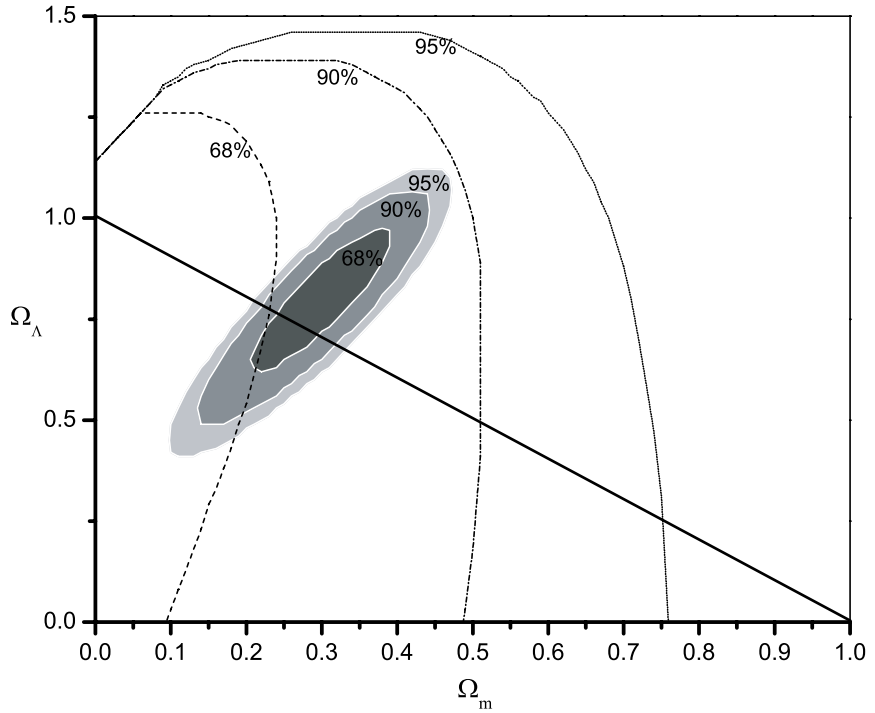

Figure 6. Constraints obtained with 30 radio galaxies alone (indicated by dashed lines) overlayed with those obtained with the combined sample of 192 supernovae and 30 radio galaxies (indicated by solid contours) in a model that allows for space curvature, nonrelativistic matter, and a cosmological constant.

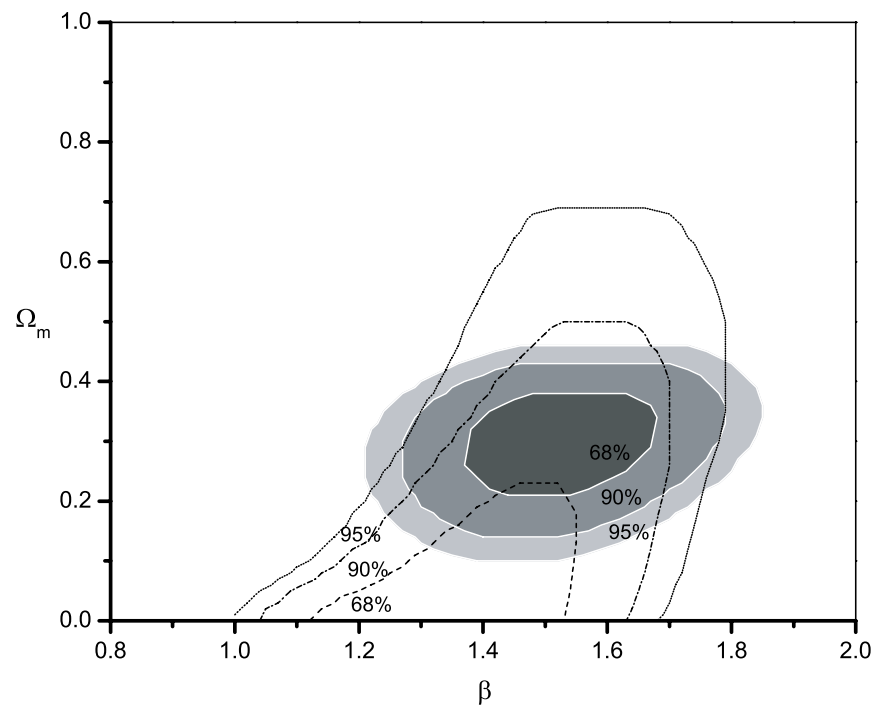

Figure 7. As in Figure 6 for the parameters $\beta$ and $\Omega_{m}$.

radio galaxies alone are overlayed on those obtained with the combined sample of supernovae and radio galaxies, which are quite similar to those obtained with supernovae alone. The radio galaxy results are consistent with but weaker than those obtained with supernovae alone; radio galaxies alone indicate that the universe is accelerating today with about $90 \%$ confidence. The fact that consistent results are obtained with two completely independent methods applied to sources with similar redshifts suggests that systematic errors are not a major problem for either method. The value of $w$ obtained with radio galaxies alone is slightly larger than -1 , the best-fit values for the supernovae samples are slightly less than -1 , and the best-fit values for the combined samples are slightly larger than -1 ; apparently the radio galaxies tend to pull the supernovae samples to slightly larger values of $w$. Again, the radio galaxy parameter $\beta$ has no covariance with cosmological parameters.

Results obtained in a lambda model with space curvature are shown in Figures 6-9. Radio galaxies alone constrain $\Omega_{m}$ to be less than about 0.5 at $90 \%$ confidence (see Figure 6).

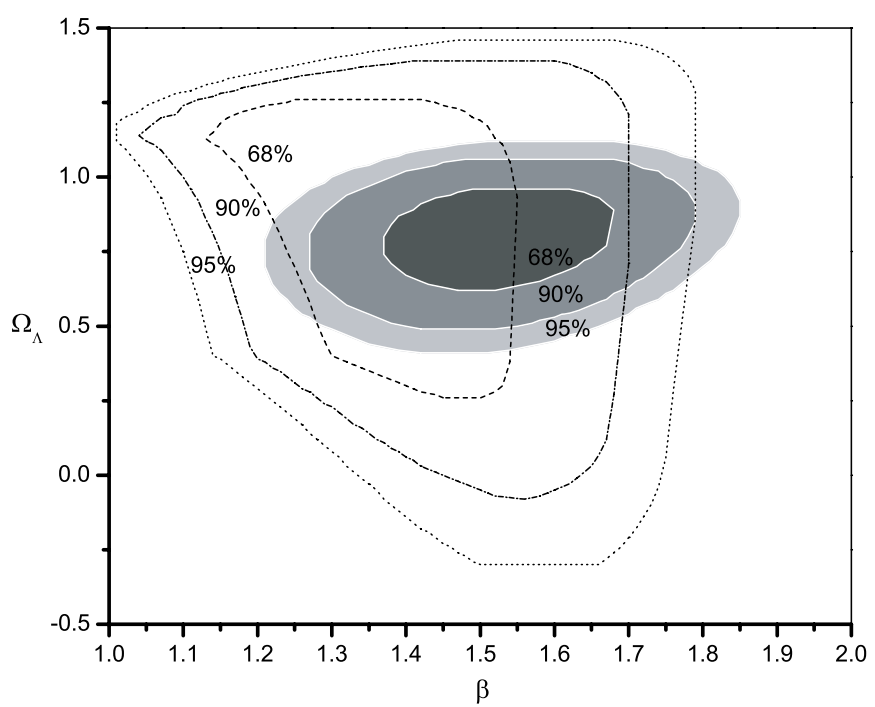

Figure 8. As in Figure 6 for the parameters $\beta$ and $\Omega_{\Lambda}$.

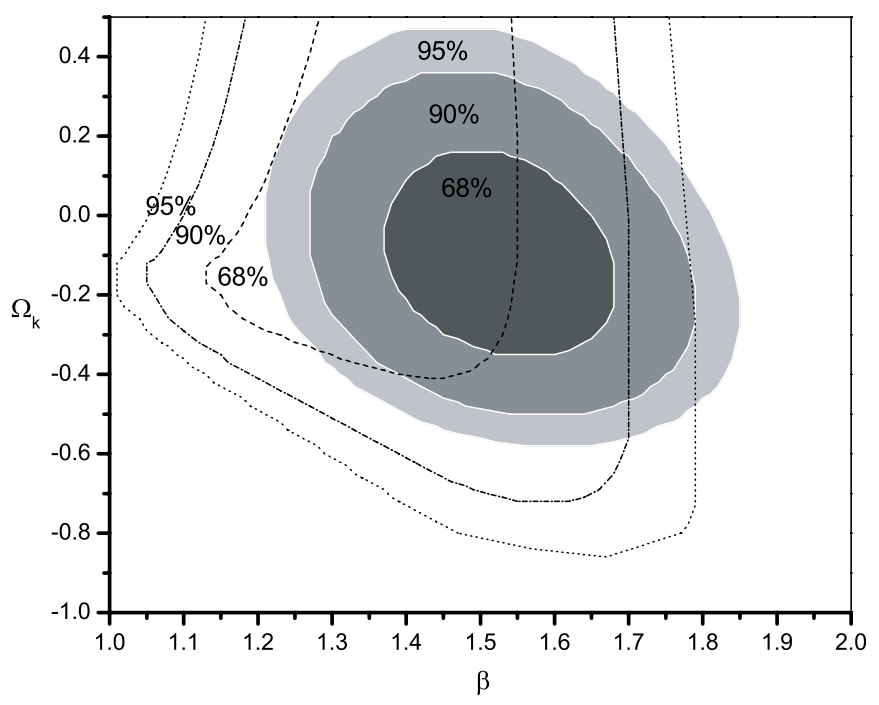

Figure 9. As in Figure 6 for the parameters $\beta$ and $\Omega_{k}$.

The combined radio galaxy and Davis et al. (2007) supernovae sample provide interesting constraints on $\beta$ as shown in Figures 7,8 , and 9 . Negative $\Omega_{k}$ indicates positive space curvature, and the results are consistent with zero space curvature. As stressed by Tegmark et al. (2006); Wright (2006), and Wang \& Mukherjee (2007), it is only by combining constraints obtained with different methods that tight constraints can be placed on space curvature; as seen here, supernovae alone and radio galaxies plus supernovae, do not place tight constraints on space curvature. Constraints on the radio galaxy model parameter $\beta$ obtained with with radio galaxies alone are overlayed on these figures, and are consistent with, though weaker than, those obtained with the full sample or with supernovae alone, suggesting that neither method is plagued by systematic errors at this level of accuracy. There is no covariance between the model parameter $\beta$ and cosmological parameters, confirming the results of Daly \& Guerra (2002). The implications of these constraints on $\beta$ are discussed in Section 2.2.

Results obtained in the rolling scalar field model of Ratra \& Peebles (1988) are shown in Figure 10. These results are significantly tighter than those reported by Samushia et al. (2007). The results obtained with the joint sample are strong, and 


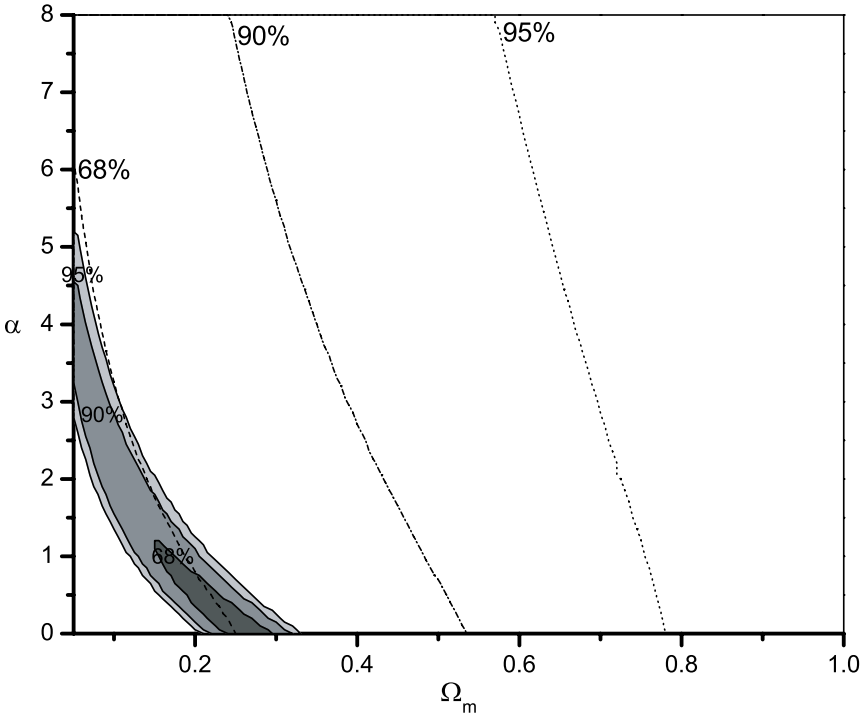

Figure 10. Constraints obtained in a spatially flat rolling scalar field model with 30 radio galaxies alone (dashed lines) overlayed with those obtained with the combined sample of 192 supernovae and 30 radio galaxies (solid contours) on the model parameter $\alpha$ and $\Omega_{m}$.

suggest that $\alpha$ is close to zero, that is, a cosmological constant provides a good description of the data.

\subsection{Implications of $\beta=1.5$}

Constraints on the radio galaxy model parameter $\beta$ indicate that there is little covariance between $\beta$ and cosmological parameters, and the value of $\beta$ is insensitive to the cosmological model. By combining the radio galaxy and supernovae data, $\beta$ can be more tightly constrained. It is very interesting indeed that for the joint fits $\beta=1.5 \pm 0.15$. This is a very special value of $\beta$, as discussed by Daly \& Guerra (2002), and may explain why these sources provide such an accurate cosmology tool.

The equations of Blandford (1990), $L_{j} \propto(a / m)^{2} B^{2} M^{2}$ for the beam power and $E_{*} \propto(a / m)^{2} M$, where $B$ is the magnetic field strength, $a$ is the spin angular momentum per unit mass, $m$ is the gravitational radius, and $M$ is the mass of the black hole that powers the outflow with beam power $L_{j}$ and total energy $E_{*}$. These are combined with the empirically derived relationship $E_{*}=L_{j} t_{*} \propto L_{j}^{1-\beta / 3}$ suggested by Daly (1994) to obtain the magnetic field strength $B \propto M^{(2 \beta-3) / 2(3-\beta)}(a / m)^{\beta /(3-\beta)}$ (Daly $\&$ Guerra 2002). When $\beta=1.5$, this simplifies to $B \propto(a / m)$, so the magnetic field strength depends only upon the spin angular momentum per unit mass and the gravitational radius of the black hole, and is independent of the black hole mass $M$. The fact that our empirically determined value of $\beta$ is very close to this special value is intriguing, and may suggest that some physical process is driving the magnetic field strength to some maximum or limiting value set by $(a / m)$. When the field strength reaches this value, the energy is released in the form of the jets with beam power $L_{j}$ that remains roughly constant over the outflow lifetime $t_{*}$ releasing a total energy $E_{*}$ through the directed jets.

A value of $\beta$ of 1.5 does not only imply that $B \propto(a / m)$; this, in turn, implies that, $L_{j} \propto E_{*}^{2}$, and $t_{*} \propto L_{j}^{-1 / 2}$, or $t_{*} \propto E_{*}^{-1}$. The last three relationships follow directly from the definition of $\beta, t_{*} \propto L_{j}^{-\beta / 3}$ and the relationship $E_{*}=L_{j} t_{*}$. A value of $\beta$ of 1.5 also implies that $L_{j} \propto(a / m)^{4} M^{2}$ or $L_{j} \propto B^{4} M^{2}$. The relationship naively expected for an Eddington limited system is
Table 4

Distances and Distance Moduli to 30 Radio Galaxies

\begin{tabular}{lccccccc}
\hline \hline Source & $\mathrm{z}$ & $y$ & $\sigma_{y}$ & $\mu_{D}$ & $\sigma_{\mu(D)}$ & $\mu_{R}$ & $\sigma_{\mu(R)}$ \\
\hline 3C 239 & 1.790 & 1.37 & 0.34 & 46.20 & 0.54 & 46.12 & 0.52 \\
3C 322 & 1.681 & 1.31 & 0.34 & 46.02 & 0.56 & 45.95 & 0.54 \\
3C 68.2 & 1.575 & 1.57 & 0.49 & 46.34 & 0.67 & 46.26 & 0.65 \\
3C 437 & 1.480 & 0.93 & 0.27 & 45.11 & 0.63 & 45.05 & 0.61 \\
3C 469.1 & 1.336 & 1.14 & 0.33 & 45.42 & 0.62 & 45.41 & 0.60 \\
3C 324 & 1.210 & 1.02 & 0.31 & 45.06 & 0.66 & 45.07 & 0.64 \\
3C 194 & 1.190 & 1.01 & 0.20 & 45.01 & 0.44 & 45.07 & 0.43 \\
3C 267 & 1.144 & 0.71 & 0.14 & 44.22 & 0.42 & 44.22 & 0.41 \\
3C 356 & 1.079 & 0.87 & 0.18 & 44.59 & 0.46 & 44.57 & 0.44 \\
3C 280 & 0.996 & 0.65 & 0.12 & 43.85 & 0.41 & 43.89 & 0.40 \\
3C 268.1 & 0.974 & 0.75 & 0.14 & 44.14 & 0.42 & 44.14 & 0.40 \\
3C 289 & 0.967 & 0.59 & 0.11 & 43.64 & 0.40 & 43.67 & 0.39 \\
3C 325 & 0.860 & 0.71 & 0.13 & 43.89 & 0.40 & 43.92 & 0.39 \\
3C 6.1 & 0.840 & 0.74 & 0.09 & 43.97 & 0.25 & 43.99 & 0.24 \\
3C 54 & 0.827 & 0.76 & 0.08 & 44.00 & 0.24 & 44.02 & 0.23 \\
3C 114 & 0.815 & 0.64 & 0.07 & 43.61 & 0.24 & 43.62 & 0.23 \\
3C 265 & 0.811 & 0.59 & 0.08 & 43.44 & 0.29 & 43.48 & 0.28 \\
3C 41 & 0.794 & 0.63 & 0.07 & 43.57 & 0.25 & 43.63 & 0.24 \\
3C 247 & 0.749 & 0.54 & 0.07 & 43.18 & 0.27 & 43.24 & 0.27 \\
3C 55 & 0.720 & 0.59 & 0.08 & 43.31 & 0.29 & 43.30 & 0.28 \\
3C 441 & 0.707 & 0.53 & 0.07 & 43.10 & 0.27 & 43.12 & 0.26 \\
3C 34 & 0.690 & 0.59 & 0.06 & 43.30 & 0.24 & 43.31 & 0.23 \\
3C 44 & 0.660 & 0.76 & 0.08 & 43.81 & 0.24 & 43.80 & 0.23 \\
3C 169.1 & 0.633 & 0.62 & 0.07 & 43.33 & 0.25 & 43.36 & 0.24 \\
3C 337 & 0.630 & 0.51 & 0.07 & 42.88 & 0.30 & 42.94 & 0.29 \\
3C 330 & 0.549 & 0.34 & 0.07 & 41.92 & 0.41 & 41.96 & 0.40 \\
3C 172 & 0.519 & 0.66 & 0.14 & 43.31 & 0.45 & 43.30 & 0.43 \\
3C 244.1 & 0.430 & 0.36 & 0.07 & 41.87 & 0.40 & 41.95 & 0.39 \\
3C 142.1 & 0.406 & 0.33 & 0.06 & 41.65 & 0.40 & 41.69 & 0.38 \\
3C 405 & 0.056 & 0.05 & 0.01 & 36.97 & 0.44 & 37.02 & 0.43 \\
\hline & & & & & & &
\end{tabular}

Notes. Values of $\mu_{D}$ were obtained for the radio galaxies using the best-fit values of $\kappa_{\mathrm{SN}}, \kappa_{\mathrm{RG}}$, and $\beta$ obtained from the joint 192 supernovae and 30 radio galaxy fits, using the average of the values of these parameters listed in Tables 1,2, and 3. Similarly, values of $\mu_{R}$ were obtained using the best-fit values of $\kappa_{\mathrm{SN}}, \kappa_{\mathrm{RG}}$, and $\beta$ obtained from the joint fits to 182 supernovae and 30 radio galaxies, using the average of the values listed in Tables 1, 2, and 3 .

$L_{j} \propto M$, so $t_{*} \propto E_{*} / M$, which only depends upon an efficiency factor when $E_{*} \propto M$. Thus, for an Eddington limited system, $t_{*}$ does not depend explicitly on $L_{j}$, and would require a value of $\beta$ of zero, which is quite clearly ruled out for these systems. Given that the most likely source of energy for these systems is spin energy of a rapidly rotating black hole, which could originate from the orbital energy of two black holes that merge, perhaps it is not surprising that the outflows have little to do with the Eddington luminosity (e.g., Blandford 1990).

Studies have shown a close relationship between the radio luminosity (jet power) and optical emission line luminosity (AGN ionizing luminosity; Baum \& Heckman 1989; Rawlings \& Saunders 1991; Xu et al. 1999; Willott et al. 1999). This may suggest a relationship between the jet lifetime of the source and the lifetime of the optically bright AGN. It is possible that both the jet lifetime and the lifetime of the optically bright AGN are not Eddington-limited, but have a power-law relationship to the total energy and beam power.

\section{DISTANCES AND DISTANCE MODULI TO RADIO GALAXIES}

There are several ways to obtain the dimensionless coordinate distance $y$ to each source. For supernovae, one way to is apply the best-fit value of the constant $\kappa_{\mathrm{SN}}$ obtained for the sample, 
and apply it to the relation $y=(1+z)^{-1} 10^{\left(\mu-\kappa_{\mathrm{SN}}\right) / 5}$, with the uncertainty of $y$ given by $\sigma_{y}=\left[\sigma_{\mu} y \ln (10)\right] / 5$, where $\sigma_{\mu}$ is the uncertainty in the distance modulus $\mu$ to the source, which follows from Equation (5). Similarly, one way to obtain the dimensionless coordinate distance $y$ to each radio galaxy is by applying the best-fit values of $\kappa_{\mathrm{RG}}=\ln \left(R_{*}\right)$ and $\beta$, and solving for the value of $y$ for which $\langle D\rangle / D_{*}=R_{*}$ for that source using Equation (4). The values of $y$ listed in Table 4 for 30 radio galaxies are obtained using the best-fit values of $\kappa_{\mathrm{RG}}$ and $\beta$ from the joint fits of 192 supernovae and 30 radio galaxies, where the average of the best-fit values from Tables 1 , 2 , and 3 for the relevant samples were used. These dimensionless coordinate distances are then converted to distance moduli and their uncertainties using the best-fit value of $\kappa_{\mathrm{SN}}$ for the Davis et al. (2007) sample of 192 supernovae (labeled $\mu_{D}$ and $\sigma_{\mu(D)}$ ) using Equation (5). Values of $y$ were also obtained (but are not listed) using the the best-fit values of $\kappa_{\mathrm{RG}}$ and $\beta$ for the joint 182 supernovae and 30 radio galaxy fits and converted to a distance modulus and its uncertainty using the best-fit value of $\kappa_{\mathrm{SN}}$ for the Riess et al. (2007) sample; these are listed in Table 4 and are labeled $\mu_{R}$ and $\sigma_{\mu(R)}$. The distance moduli $\mu_{D}$ for the radio galaxies can be combined with those for supernovae listed by Davis et al. (2007), and the distance moduli $\mu_{R}$ for the radio galaxies can be combined with those for the supernovae listed by Riess et al. (2007).

The values of the constants that are used to obtain the dimensionless coordinate distances are rather insensitive to the model used to obtain them; similar results are obtained in the lambda model with space space curvature, the quintessence model, and the rolling scalar field model, and average values of the constants obtained in the context of these cosmological models relevant to each sample was used to obtain the values listed in Table 4. There are two alternative ways to obtain the dimensionless coordinate distances to the supernovae and radio galaxies that do not require the use of the best-fit model parameters $\kappa_{\mathrm{SN}}, \kappa_{\mathrm{RG}}$, and $\beta$. These methods yield results very similar to those obtained using the best-fit parameters. The alternative methods start with the values of $\mu$ for supernovae and $\langle D\rangle / D_{*}$ for radio galaxies, obtain the luminosity distance or the coordinate distance $\left(a_{0} r\right)$ to each source, which is in units of Mpc, and then solve for the dimensionless coordinate distance using the equation $y=\left(H_{0} / c\right)\left(a_{0} r\right)$ by finding a way to determine $H_{0}$ that is appropriate for that sample, which often is not stated in the data papers. This value of $H_{0}$ that was input needs to be removed, and can be obtained by fitting the low redshift data to the Hubble law, or by requiring that at zero redshift the function $E(z)=H(z) / H_{0}$, described by Daly \& Djorgovski (2003; see also Daly et al. 2008), is equal to one. It turns out that the values of $y$ obtained with any of these three methods are very similar.

The dimensionless coordinate distances to the 30 radio galaxies and 192 supernovae of Davis et al. (2007) are shown in Figure 11; The supernovae dataset includes the essence supernovae presented by Wood-Vasey (2007), the legacy supernovae presented by Astier et al. (2006), and the high redshift HST supernovae presented by Riess et al. (2007).

Although the radio galaxy and supernovae methods are very different, there is good agreement between the dimensionless coordinate distances to sources at similar redshift. The supernovae distances rely on optical observations for rather short lived events, whereas the the radio galaxy distances rely on radio observations for sources that have lifetimes on the order of millions of years. The methods rely upon measurements of

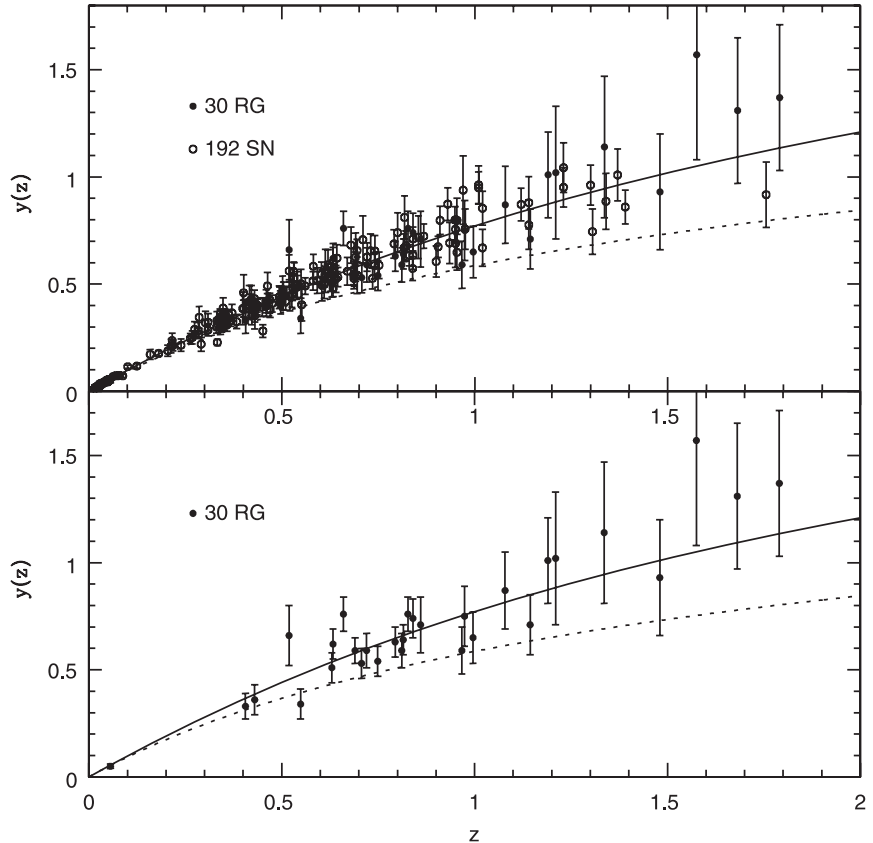

Figure 11. Dimensionless coordinate distances to 30 radio galaxies compared with those to the 192 supernovae of Davis et al. (2007). The dashed curve indicates $y(z)$ expected in a flat matter dominated universe with $\Omega_{m}=1$, and the solid curve indicates that expected in a flat lambda dominated universe with $\Omega_{m}=0.3$ and $\Omega_{\Lambda}=0.7$; note that $y(z)$ is independent of $H_{0}$. Clearly, the data are well described by a cosmological constant with $\Omega_{\Lambda}=0.7$.

entirely different quantities that are then applied using completely different astrophysical arguments. Thus, any systematic effects are likely to be quite different for the two types of sources. The fact that they yield quite similar results is encouraging.

\section{SUMMARY}

A sample of 30 radio galaxies was used to determine cosmological parameters and the radio galaxy model parameters $\kappa_{\mathrm{RG}}$ and $\beta$ in three standard cosmological models. Nearly identical values of $\kappa_{\mathrm{RG}}$ and $\beta$ are obtained in each cosmological model indicating that they are not strongly affected by the context in which they are determined (see Tables 1, 2, and 3), thus they can be used to determine the dimensionless coordinate distance to each source.

Three supernovae samples are considered both separately and jointly with the radio galaxy sample and are analyzed in the context of three standard cosmological models (see Tables 1, 2 , and 3) to determine cosmological parameters and the model parameter $\kappa_{\mathrm{SN}}$. Nearly identical values of $\kappa_{\mathrm{SN}}$ are obtained for each supernovae sample in the context of each cosmological model, thus they can be used to determine the dimensionless coordinate distance to each source. They can also be used to determine the effective distance modulus to each of the radio galaxies, which can then be combined with those of the supernovae to increase the sample sizes, particularly at high redshift.

Constraints on cosmological parameters obtained with radio galaxies are consistent with, though weaker than, those obtained with supernovae alone. There are no inconsistencies between results obtained with radio galaxies and supernovae. For example, in the context of a standard quintessence model, radio galaxies alone indicate that the universe is accelerating today with about 90\% confidence (see Figure 6). The consistency between re- 
sults obtained with radio galaxies alone, supernovae alone, and the combined supernovae and radio galaxy samples suggests that neither method is plagued by unknown systematic errors; both methods seem to be working well. The radio galaxy and supernovae methods are completely independent, based on a completely different physics and observations, and provide independent measures of distances to sources at similar redshift. The facts that the cosmological parameters obtained in specific models are consistent, and that the coordinate distances to sources at similar redshift are consistent suggests that systematic errors are not playing a major role in either method.

Since nearly identical values of $\kappa_{\mathrm{SN}}, \kappa_{\mathrm{RG}}$, and $\beta$ are obtained in each cosmological model, they can be used to solve for the dimensionless coordinate distance to each source, $y$. Values of $y$ to each radio galaxy, obtained using the best-fit values of $\kappa_{\mathrm{RG}}$ and $\beta$ indicated by fits to the joint sample of 192 supernovae and 30 radio galaxies, are listed in Table 4 . The best-fit values of $y$ listed in Table 4 are combined with the best-fit value of $\kappa_{\mathrm{SN}}$ obtained for the Davis et al. (2007) sample to obtain the distance modulus to each of the radio galaxies, $\mu_{D}$, which are listed in Table 4 and which can be added to those already listed in Davis et al. (2007). Similarly, the values of $y$ to the radio galaxies obtained using the best-fit values of $\kappa_{\mathrm{RG}}$ and $\beta$ indicated by fits to the joint sample of 182 supernovae and 30 radio galaxies were obtained (but are not listed), and were combined with the best-fit value of $\kappa_{\mathrm{SN}}$ obtained for the Riess et al. (2007) sample to obtain values of $\mu_{R}$ for the radio galaxies that can be combined with those already listed by Riess et al. (2007). Since nearly identical values of $\kappa_{\mathrm{SN}}, \kappa_{\mathrm{RG}}$, and $\beta$ and their uncertainties are obtained in each cosmological model, the average of the values obtained in the different cosmological models and their uncertainties were used.

New constraints were obtained on the model parameter $\alpha$ in the rolling scalar field model of Peebles \& Ratra (1988). These constraints are rather strong and indicate that $\alpha$ is close to zero for reasonable values of $\Omega_{m}$. Thus, a cosmological constant provides a good description of the data, and there is no indication that the energy density of the dark energy is changing with redshift.

New constraints were obtained on the radio galaxy model parameter $\beta$, suggesting values of $\beta$ close to 1.5 . This is interpreted in the context of a standard magnetic braking model of energy extraction from a rotating black hole (e.g. Blandford 1990). This is a very special value of $\beta$ for which the braking magnetic field strength depends only upon the spin angular momentum per unit mass and the gravitational radius of the black hole. This suggests that when the magnetic field strength reaches this maximum or limiting value, the relativistic outflow is triggered. The fact that the magnetic field strength does not depend explicitly on the black hole mass for this special value of $\beta$ may explain why it is that this paricular type of radio source is able to provide a modified standard yardstick for cosmological studies.

We have provided further evidence that radio galaxies can be used to determine coordinate distances to sources, and thus cosmological parameters through a standard angular diameter test. Our comparative study shows that values of cosmological parameters obtained with radio galaxies are consistent with those obtained with supernovae. Supernovae alone and radio galaxies alone both indicate that the universe is accelerating at the current epoch when these data are analyzed in specific models such as a quintessence model in spatially flat universe, a lambda model in universe that allows for nonzero space curvature, and a rolling scalar field model in a spatially flat universe. All of these models rely upon the equations of general relativity, and the results obtained in these models would not be correct if general relativity is not the correct theory of gravity. These data are analyzed in a model-independent way that does not rely upon general relativity by Daly et al. (2008), who show that the supernovae data alone and the radio galaxy data alone indicate that the universe is accelerating at the current epoch independent of whether general relativity is the correct theory of gravity. When expressed as coordinate distances, both radio galaxy and supernova samples can be combined and used in a joint cosmological analysis, as it was done, e.g., by Daly \& Djorgovski (2003, 2004). We use these expanded and combined samples in a separate paper (Daly et al. 2008).

We thank the observers for their tireless efforts in obtaining the data used for this study. We also thank the referee for providing very helpful suggestions on this work. This work was supported in part by U. S. National Science Foundation grants AST-0507465 (R.A.D.) and AST-0407448 (S.G.D.), and the Ajax Foundation (S.G.D.).

\section{REFERENCES}

Alexander, P. 1987, MNRAS, 225, 27

Alexander, P., \& Leahy, J. P. 1987, MNRAS, 225, 1

Antonucci, R. 1993, ARA\&A, 31, 473

Astier, P., et al. 2006, A\&A, 447, 31

Barai, P., \& Wiita, P. J. 2006, MNRAS, 372, 381

Barai, P., \& Wiita, P. J. 2007, ApJ, 658, 217

Baum, S. A., \& Heckman, T. 1989, ApJ, 336, 702

Begelman, M. C., Blandford, R. D., \& Rees, M. J. 1984, Rev. Mod. Ph., 56, 255

Begelman, M. C., \& Cioffi, D. F. 1989, ApJ, 345, L21

Blandford, R. D. 1990, in Active Galactic Nuclei, ed. T. J. L. Courvoisier \& M. Mayor (Berlin: Springer), 161

Blandford, R. D., \& Rees, M. J. 1974, MNRAS, 169, 395

Buchalter, A., Helfand, D. J., Becker, R. H., \& White, R. L. 1998, ApJ, 494, 503

Chen, G., \& Ratra, B. 2003, ApJ, 582, 586

Condon, J. J. 1984a, ApJ, 284, 44

Condon, J. J. 1984b, ApJ, 287, 461

Daly, R. A. 1990, ApJ, 355, 416

Daly, R. A. 1994, ApJ, 426, 38

Daly, R. A., \& Djorgovski, S. G. 2003, ApJ, 597, 9

Daly, R. A., \& Djorgovski, S. G. 2004, ApJ, 612, 652

Daly, R. A., Djorgovski, S. G., Freeman, K. A., Mory, M. P., O’Dea, C. P., Kharb, P., \& Baum, S. 2008, ApJ, 677, 1

Daly, R. A., \& Guerra, E. J. 2002, AJ, 124, 1831

Davis, T. M., et al. 2007, ApJ, 666, 716

De Young, D. S. 2002, The Physics of Extragalactic Radio Sources (Chicago, IL: Univ. of Chicago Press)

Dunlop, J. S., \& Peacock, J. A. 1990, MNRAS, 247, 19

Fanaroff, B. L., \& Longair, M. D. 1972, MNRAS, 159, 119

Gopal-Krishna, \& Wiita, P. J. 1987, MNRAS, 226, 531

Guerra, E. J., \& Daly, R. A. 1998, ApJ, 493, 536

Guerra, E. J., Daly, R. A., \& Wan, L. 2000, ApJ, 544, 659

Gurvits, L. I., Kellerman, K. I., \& Frey, S. 1999, A\&A, 342, 378

Hoyle, F., \& Burbidge, G. R. 1970, Nature, 227, 359

Jackson, J. C. 2004, JCAP, 11, 7

Jackson, J. C., \& Jannetta, A. L. 2006, JCAP, 11, 2

Jamrozy, M. 2004, A\&A, 419, 63

Kaiser, C. R., \& Alexander, P. 1999, MNRAS, 302, 515

Kapahi, V. K. 1975, MNRAS, 172, 513

Kapahi, V. K. 1985, MNRAS, 214, 19

Kayser, R. 1995, A\&A, 294, 21

Kellerman, K. I. 1972, AJ, 77, 531

Kellerman, K. I. 1993, Nature, 361, 134

Kharb, P., O’Dea, C. P., Baum, S. A., Daly, R., Mory, M., Donahue, M., \& Guerra, E. 2008, ApJS, 174, 74

Laing, R. A., Riley, J. M., \& Longair, M. S. 1983, MNRAS, 204, 151 
Leahy, J. P. 1991, in Beams and Jets in Astrophysics, ed. P. A. Hughes (Cambridge: Cambridge Univ. Press)

Leahy, J. P., Muxlow, T. W. B., \& Stephens, P. W. 1989, MNRAS, 239, 401

Leahy, J. P., \& Williams, A. G. 1984, MNRAS, 210, 929

Longair, M. S. 1976, Nature, 263, 372

Longair, M. S., \& Pooley, G. G. 1969, MNRAS, 145, 121

O’Dea, C. P., Daly, R., Kharb, P., Freeman, K. A., \& Baum, S. A. 2008, A\&A, in press

Pelletier, G., \& Roland, J. 1989, A\&A, 224, 24

Podariu, S., Daly, R. A., Mory, M. P., \& Ratra, B. 2003, ApJ, 584, 577

Rawlings, S. 2002, in Proc. IAU Symp. 199, The Universe at Low Radio Frequencies, ed. A. P. Rao, G. Swarup, \& Gopal Krishna (San Francisco, CA: ASP), 34

Rawlings, S., \& Saunders, R. 1991, Nature, 349, 138

Ratra, B., \& Peebles, P. J. E. 1988, Phys. Rev. D, 37, 3406

Ratra, B., \& Vogeley, M. S. 2008, PASP, 120, 235
Readhead, A. C. S., \& Longair, M. S. 1975, MNRAS, 170, 393

Rees, M. J. 1972, in IAU Symp. 44, External Galaxies and Quasi-Stellar Objects, ed. D. S. Evans, D. Wills, \& B. J. Wills (Dordrecht: Reidel), 407

Riess, A. G., et al. 2007, ApJ, 659, 98

Rowan-Robinson, M. 1967, Nature, 216, 1289

Samushia, L., Chen, G., \& Ratra, B. 2007, arXiv:0706.1963

Scheuer, P. A. G. 1974, MNRAS, 166, 513

Singal, A. K. 1993, MNRAS, 263, 139

Tegmark, M., et al. 2006, Phys. Rev. D, 74, 123507

Wall, J. V., Pearson, T. J., \& Longair, M. S. 1980, MNRAS, 193, 683

Wang, Y., \& Mukherjee, 2007, Phys. Rev. D, 76, 103533

Willott, C., Rawlings, S., Blundell, K. M., \& Lacy, M. 1999, MNRAS, 309, 1017

Wood-Vasey, W. M., et al. 2007, ApJ, 666, 694

Wright, E. L. 2006, astro-ph/0603750

Xu, C., Livio, M., \& Baum, S. A. 1999, AJ, 118, 1169 